\title{
$\underline{\mathbf{P}-202}$
}

\section{Virgin Coconut oil (VCO) Inhibits Cell Growth Via Apoptosis on Lung Cancer Cell Lines}

\author{
Kamalaldin $\mathrm{NA}^{1}$, Sulaiman $\mathrm{SA}^{2}$, Seeni $\mathrm{A}^{3}$ and Yahaya $\mathrm{BH}^{1}$ \\ ${ }^{I}$ Cluster for Regenerative Medicine, Advanced Medical and Dental Institute, Universiti Sains Malaysia, 13200 Kepala \\ Batas, Penang, Malaysia; ${ }^{2}$ Department of Pharmacology, School of Medical Sciences, Universiti Sains Malaysia, 16150 \\ Kubang Kerian, Kelantan, Malaysia; ${ }^{3}$ Cluster of Toxicology, Advanced Medical and Dental Institute, Universiti Sains \\ Malaysia, 13200 Kepala Batas, Penang, Malaysia
}

Virgin coconut oil (VCO) is rich with antioxidant and exhibit anti-cancerous properties. The ability of VCO to inhibit the growth of lung cancer cells by inducing apoptosis has yet been reported. NCI-H1299 (a non-small cancer cell) and A549 (adenocarcinomic human alveolar basal epithelial cells) were incubated in media containing various concentration of VCO for $1,3,5$ and 8 days. The morphology of the cells were observed and viable cells was counted following treatments. Apoptotic features were observed on cell membrane treated with VCO, indicated by the appeareance of giant bubbles and massive cytoplasmic vacuoles. The effect of VCO treatment also was observed in cell proliferation assay in which high doses of VCO greatly inhibit the cell proliferation activity as early as 1 day post treatment and suggest it was a time-dependent. This study shown that VCO can induce apoptosis in cancer cell lines which responsible for the anti-cancer properties.

\section{ACKNOWLEDGEMENTS}

ScienceFund Grant 305/CIPPT/613224, Ministry of Science, Technology and Innovation and Universiti Sains Malaysia. 